\title{
A GNAS1 imprinting defect in pseudohypoparathyroidism type IB
}

\author{
Jie Liu, ${ }^{1}$ Deborah Litman, ${ }^{1}$ Marjorie J. Rosenberg, ${ }^{2}$ Shuhua Yu, ${ }^{1}$ Leslie G. Biesecker, ${ }^{2}$ \\ and Lee S. Weinstein ${ }^{1}$ \\ ${ }^{1}$ Metabolic Diseases Branch, National Institute of Diabetes and Digestive and Kidney Diseases, and
${ }^{2}$ Genetic Disease Research Branch, National Human Genome Research Institute, NIH, Bethesda, Maryland, USA
}

Address correspondence to: Lee S. Weinstein, Metabolic Diseases Branch, National Institute of Diabetes and Digestive and Kidney Diseases, National Institutes of Health, Building 10, Room 8C101, Bethesda, Maryland 20892-1752, USA. Phone: (301) 402-2923; Fax: (301) 402-0374; E-mail: leew@amb.niddk.nih.gov.

Received for publication May 25, 2000, and accepted in revised form September 19, 2000.

\begin{abstract}
Pseudohypoparathyroidism type IB (PHPIB) is characterized by renal resistance to parathyroid hormone (PTH) and the absence of other endocrine or physical abnormalities. Familial PHPIB has been mapped to $20 \mathrm{q} 13$, near GNAS1, which encodes $\mathrm{G}_{\mathrm{s}} \alpha$, the $\mathrm{G}$ protein $\alpha$-subunit required for receptorstimulated cAMP generation. However, $\mathrm{G}_{\mathrm{s}} \alpha$ function is normal in blood cells from PHPIB patients, ruling out mutations within the $G_{s} \alpha$ coding region. In mice $G_{s} \alpha$ is expressed only from the maternal allele in renal proximal tubules (the site of PTH action) but is biallelically expressed in most other tissues. Studies in patients with Albright hereditary osteodystrophy suggest a similar $\mathrm{G}_{\mathrm{s}} \alpha$ imprinting pattern in humans. Here we identify a region upstream of the $\mathrm{G}_{s} \alpha$ promoter that is normally methylated on the maternal allele and unmethylated on the paternal allele, but that is unmethylated on both alleles in all 13 PHPIB patients studied. Within this region is an alternative promoter and first exon (exon 1A), generating transcripts that are normally expressed only from the paternal allele, but that are biallelically expressed in PHPIB patients. Therefore, PHPIB is associated with a paternal-specific imprinting pattern of the exon $1 \mathrm{~A}$ region on both alleles, which may lead to decreased $\mathrm{G}_{\mathrm{s}} \alpha$ expression in renal proximal tubules. We propose that loss of exon $1 \mathrm{~A}$ imprinting is the cause of PHPIB.
\end{abstract}

J. Clin. Invest. 106:1167-1174 (2000).

\section{Introduction}

Pseudohypoparathyroidism type IB (PHPIB) is defined by renal resistance to parathyroid hormone (PTH) in the absence of other endocrine or physical abnormalities. In PHPIB, urinary cAMP responses to administered PTH are blunted (1), implicating a defect in the signaling pathway proximal to cAMP generation (e.g., PTH receptor or the $G$ protein $G_{s}$ ). PHPIB is usually sporadic, but occasionally is familial. Several lines of evidence $(2,3)$, including its mapping to $20 \mathrm{q} 13$ in four families (4), have ruled out mutations in the PTHreceptor locus as the cause of PHPIB. One gene in the $20 \mathrm{q} 13$ region is GNAS1, which encodes $\mathrm{G}_{\mathrm{s}} \alpha$, the G-protein $\alpha$-subunit required for receptor-stimulated cAMP generation (5). However, $\mathrm{G}_{\mathrm{s}} \alpha$ expression and function are normal in peripheral blood cells from PHPIB patients $(1,6)$, ruling out inactivating mutations within the $\mathrm{G}_{\mathrm{s}} \mathrm{\alpha}$-coding regions as the cause of PHPIB.

GNAS1 is a complex gene that encodes multiple products by use of four alternative first exons that splice onto a common set of downstream exons $(5,7-10)$ (see details in Figure 1a). The most downstream promoter (exon 1) produces transcripts encoding $\mathrm{G}_{\mathrm{s}} \alpha(5,7)$. Alternative GNAS1 promoters located 35 and $47 \mathrm{~kb}$ upstream of exon 1 produce transcripts encoding $\mathrm{XL} \alpha \mathrm{s}$, a Golgi-specific isoform of $\mathrm{G}_{\mathrm{s}} \alpha$, and the chro- mogranin-like protein NESP55, respectively $(8,9,11)$. Both of these proteins are expressed primarily in neuroendocrine tissues, and little is known about their biological functions. A fourth alternative first exon (exon $1 \mathrm{~A})$, located $2.5 \mathrm{~kb}$ upstream of $\mathrm{G}_{\mathrm{s}} \alpha$ exon 1 , generates transcripts of unknown function $(12,13)$.

Another layer of complexity is the observation that the GNAS1 promoter regions are imprinted. Genomic imprinting is an epigenetic phenomenon characterized by expression from a single parental allele and is often associated with methylation of the promoter (at cytidines within CPG dinucleotides) on the inactive allele (14-16). The XLos and NESP55 promoters are oppositely imprinted: XLos is expressed from the paternal allele and its promoter region is methylated on the maternal allele, whereas NESP55 is expressed from the maternal allele and its promoter is methylated on the paternal allele (8, $9,11)$. We have recently shown in mice that exon $1 \mathrm{~A}$ is expressed only from the paternal allele and that its promoter is methylated on the maternal allele (10).

Clinical studies suggest that $\mathrm{G}_{\mathrm{s}} \alpha$ is imprinted in a tissue-specific manner in humans. Heterozygous GNAS1 mutations within exons 1-13 (see Figure 1a) that disrupt $G_{s} \alpha$ expression or function lead to Albright hereditary osteodystrophy (AHO), a syndrome characterized by obesity, short stature, brachy- 
dactyly (shortening of metacarpals and metatarsals), subcutaneous ossifications, and mental deficits (17-19). Paternal transmission of such mutations results in only the AHO phenotype (pseudopseudohypoparathyroidism [PPHP]), whereas maternal transmission produces offspring who also have resistance to PTH and minimal thyrotropin resistance (pseudohypoparathyroidism type IA [PHPIA]) $(5,20,21)$. If $G_{s} \alpha$ is expressed only from the maternal allele in hormone target tissues (e.g., renal proximal tubules, the site of PTH action), then mutations in the maternal allele will disrupt hormone signaling while mutations in the paternal allele will have minimal effects. This plausibly explains why PTH-stimulated urinary cAMP responses are markedly blunted in PHPIA but are unaffected in PPHP (20). $\mathrm{G}_{\mathrm{s}} \alpha$ imprinting would have to be tissue specific, because $G_{s} \alpha$ is biallelically expressed in lymphocytes (8) and fetal tissues (22), and both PHPIA and PPHP patients have similar (approximately $50 \%$ ) reductions in $\mathrm{G}_{s} \alpha$ expression in erythrocytes (20). Indeed, in mice $\mathrm{G}_{\mathrm{s}} \alpha$ is maternally expressed in some tissues (including the renal proximal tubules), but is biallelically expressed in most other tissues (23, 24). However, definitive evidence for the tissue-specific imprinting of $\mathrm{G}_{\mathrm{s}} \alpha$ in humans is still lacking $(8,22)$.

The mechanisms by which $\mathrm{G}_{\mathrm{s}} \alpha$ is imprinted are unknown. $G_{s} \alpha$ imprinting is not secondary to silencing of its promoter by methylation because the $G_{s} \alpha$ promoter is unmethylated on both alleles $(8,10,11)$. We showed recently that an approximately $2.5-\mathrm{kb}$ region located approximately $1-3.5 \mathrm{~kb}$ upstream of the mouse $\mathrm{G}_{\mathrm{s}} \alpha$ translational start site is methylated exclusively on the maternal allele, and that the exon 1A promoter, which is located within this differentially methylated region (DMR), is active only on the paternal allele (10). Allele-specific methylation of this region is established during gametogenesis and is present in all somatic tissues, suggesting that it may be important for the establishment of imprinting in the orthologous mouse gene Gnas. We hypothesize that the exon 1A DMR may be important for establishing and/or maintaining the tissue-specific imprinting of $G_{s} \alpha$. If this is true, then paternal-specific imprinting of the exon 1A DMR on both alleles would reduce $G_{s} \alpha$ expression in renal proximal tubules (which normally express $G_{s} \alpha$ only from the maternal allele), but have little effect on $\mathrm{G}_{\mathrm{s}} \alpha$ expression in other tissues (which normally express $G_{s} \alpha$ equally from both parental alleles). Such an exon $1 \mathrm{~A}$ imprinting defect could cause the isolated renal PTH resistance characteristic of PHPIB.

In this paper we first show that the human exon 1A-promoter region is imprinted in a manner similar to that in the mouse. We then demonstrate that in all 13 PHPIB patients studied, this region has a paternalspecific imprinting pattern on both alleles. In contrast, imprinting of the XL $\alpha$ s- and NESP55-promoter regions was abnormal in only a subset of PHPIB patients. Thus, we have identified exon $1 \mathrm{~A}$, a new imprinted region within GNAS1, that is abnormally imprinted in PHPIB. We propose that the exon 1A $D M R$ is important for $G_{s} \alpha$ imprinting and that loss of exon $1 \mathrm{~A}$ imprinting is the cause of PHPIB.

\section{Methods}

Patients. All PHPIB patients initially presented with hypocalcemia, hyperphosphatemia, and elevated serum PTH levels in the absence of renal insufficiency or any of the clinical or radiological features of AHO. Serum thyrotropin (TSH), thyroxine (T4), free T4, triiodothyronine (T3), and 25-hydroxyvitamin D levels were normal in all patients. Two patients (patients 5 and 10) had overt osteitis fibrosa cystica at presentation that resolved after instituting oral calcium and vitamin D therapy. Two other patients (patients 3 and 4) had at least one other affected family member. Informed consent was obtained from all patients and normal subjects and the research protocol was approved by the National Institute of Arthritis and Musculoskeletal and Skin Diseases/National Institute of Diabetes and Digestive and Kidney Diseases institutional review board.

Southern analysis. Genomic DNA was isolated from patient blood samples using the Blood \& Cell Culture DNA Maxi kit (QIAGEN, Valencia, California, USA). DNA samples $(20 \mu \mathrm{g})$ were digested with the indicated restriction enzymes (New England Biolabs, Beverly, Massachusetts, USA), separated by electrophoresis on a $1.5 \%$ agarose gel, and transferred to nylon filters (Nytran; Schleicher \& Schuell, Keene, New Hampshire, USA). Genomic probes were generated by PCR using the following sets of upstream and downstream primers: $5^{\prime}$ GCTTGCCGCTTGCTCCTTGCCAC-3' and $5^{\prime}$-TTCGCAACTTGAGAGCGTGCAGAC-3' for the NESP55-promoter region (9); 5'-TGCTGAGACCCGGGCAGCCCATGT-3' and 5'-CTGTCTGagtCCAaGCAGCCGTGCCAGG-3' for the XLos-promoter region (8); and 5'-GTGAGCCGCGGAACTGGCAGGCA-3' and 5'-GCTGGGCTGGCTGCCTAAGAG-3' for the exon 1A region. Probes were labeled with ${ }^{32} \mathrm{P}$ by random priming using the Multiprime DNA Labeling kit (Amersham Pharmacia Biotech, Arlington Heights, Illinois, USA). Filters were incubated with probes in QuikHyb hybridization solution (Stratagene, La Jolla, California, USA) at $68^{\circ} \mathrm{C}$ for 1 hour and then washed twice with $2 \times \mathrm{SSC}(1 \times \mathrm{SSC}$ is $0.15 \mathrm{M} \mathrm{NaCl}$ plus $0.015 \mathrm{M}$ sodium citrate), $0.1 \%$ (wt/vol) SDS for $30 \mathrm{~min}-$ utes at room temperature, and once with $0.1 \times$ SSC, $0.1 \%$ (wt/vol) SDS for 1 hour at $60^{\circ} \mathrm{C}$. Filters were exposed to Bio-Max MR films (Eastman Kodak Co., Rochester, New York, USA).

To rule out deletion of the exon $1 \mathrm{~A}$ region quantitative Southern analysis was performed by digesting genomic DNA samples from normal and PHPIB patients using SacI. After electrophoresis and blotting, the XL $\alpha$ s probe was hybridized to the $6.0-\mathrm{kb}$ band (see Figure $1 \mathrm{~b}$ ), and an exon $1 \mathrm{~A}$ probe generated by PCR using the primers $5^{\prime}$-TTCCCCTTCGGTTTATAGGGGCCGCTG-3' and 5'-TGGCAGGAGTCTGTTTACCCTC-3' was hybridized to a $2.6-\mathrm{kb}$ band that includes the exon $1 \mathrm{~A}$ 
region. The 6.0-kb XLos-specific and 2.6-kb exon $1 \mathrm{~A}-$ specific bands in each sample were quantified using a phosphorimager (BAS-1500; Fuji Medical Systems, Stamford, Connecticut, USA).

Genotyping. We identified a 5-bp insertion polymorphism within exon 1A. To genotype patients and normal subjects for the 5-bp insertion polymorphism, genomic DNA was amplified by PCR using primers upstream (5'-TTCCCCTTCGGGGGGCCGCTGC-3') and downstream (5'-TGGCAGGAGTCTGTTTACCCTC- $\left.3^{\prime}\right)$ of the polymorphic site, and the products were sequenced directly using the THERMO Sequenase kit (Amersham Pharmacia Biotech) and the upstream primer. To determine which parental allele is normally methylated within the exon 1A region, genomic DNA from normal sub- jects heterozygous for the polymorphism was digested with PstI and AscI and separated on a $0.8 \%$ agarose gel. Fractions containing the $2.8-\mathrm{kb}$ methylated and $1.6-\mathrm{kb}$ unmethylated fragments (see Figure 1b) were excised from the gel and purified using the QIAEX II kit (QIAGEN). The fractions containing the methylated and unmethylated alleles were individually genotyped by PCR and direct sequencing, as described above. For parental assignment, the sequences were run next to the sequence obtained from one homozygous parent.

Bisulfite-modified genomic sequencing. Genomic DNA isolated from normal blood samples was analyzed for methylation of the exon $1 \mathrm{~A}$ region by bisulfite-modified genomic sequencing as previously described (10) using the following sets of upstream and downstream
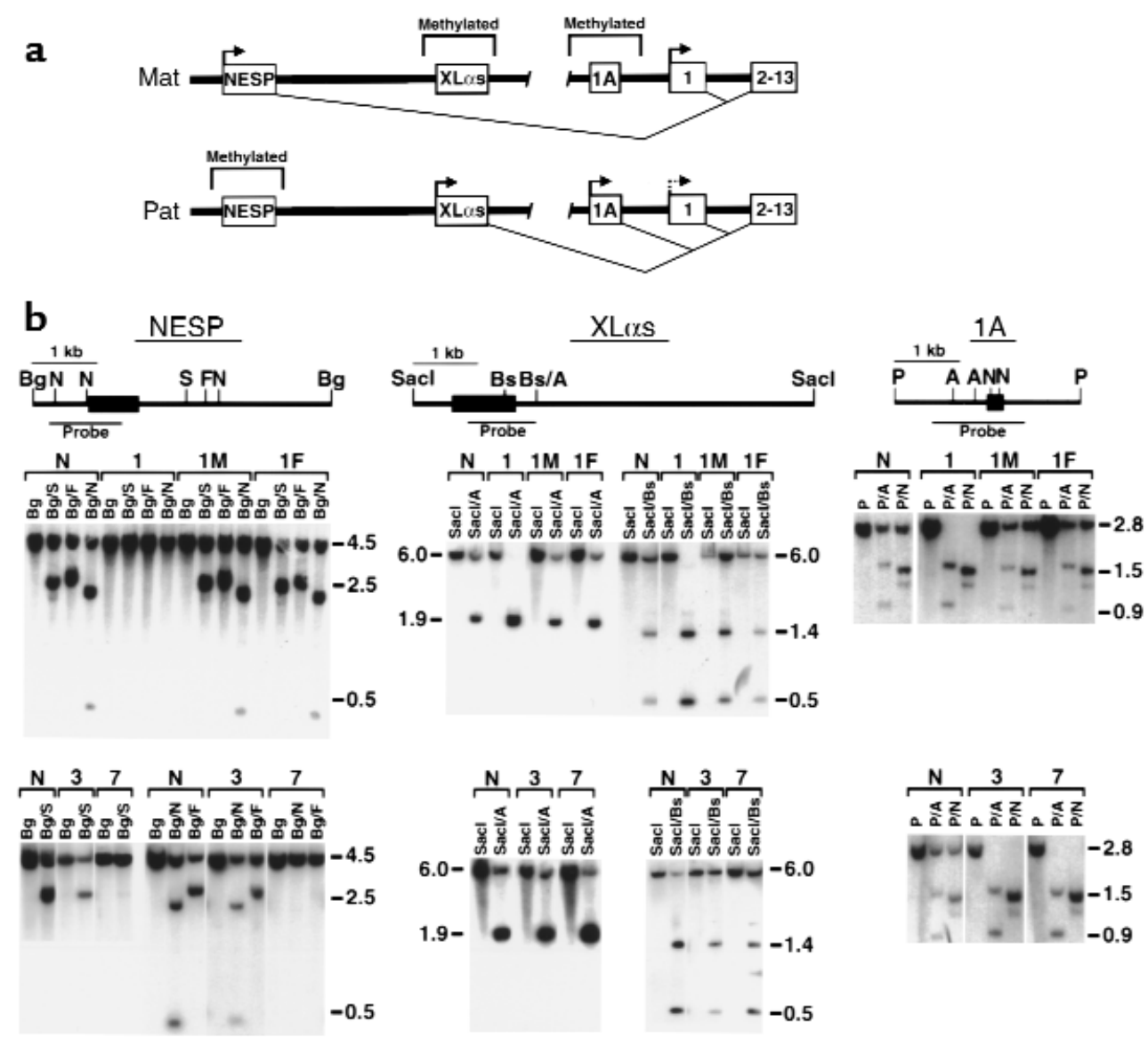

Figure 1

Methylation analysis of GNAS1. (a) The normal allele-specific methylation and expression patterns of the four known first exons of GNAS1, which splice onto exon 2 to produce transcripts encoding NESP55, XL $\alpha$ s, a transcript of unknown function (exon $1 A)$, and $\mathrm{G}_{\mathrm{s}} \alpha($ exon 1$)$. Horizontal arrows indicate transcriptionally active promoters. The imprinting of NESP55 and XL $\alpha$ s have been defined previously ( 8,9 , 11). Exon 1 is probably paternally imprinted in some tissues, indicated by the dashed arrow. NESP55 protein is unrelated to $\mathrm{G}_{\mathrm{s}} \alpha$, and its entire coding region is located within its first exon. In contrast, $\mathrm{XL} \alpha \mathrm{s}$ and $\mathrm{G}_{\mathrm{s}} \alpha$ proteins have identical $\mathrm{COOH}$-terminal domains (encoded by exons 2-13), while their unique $\mathrm{NH}_{2}$-terminal domains are encoded within their respective first exons. Exon $1 \mathrm{~A}$ does not have a translational start site, and its transcripts are likely to be untranslated. The imprinting of the exon $1 \mathrm{~A}$ region has been defined previously in mice (10). (b) Southern analyses of leukocyte genomic DNA from a normal subject (N), PHPIB patients 1, 3, and 7, the mother of patient $1(1 \mathrm{M})$, and the father of patient $1(1 \mathrm{~F})$, using genomic DNA probes from the NESP55 (left panel; ref. 9), XL $\alpha$ s (middle panel; ref. 8), and exon $1 \mathrm{~A}$ (right panel) regions. Above are the relevant restriction maps depicting each upstream exon as a black box and the position of the probes below. Patient 1 is abnormally methylated in all three regions, patient 3 is abnormally methylated only in the exon $1 \mathrm{~A}$ region, and patient 7 is abnormally methylated in the NESP55 and exon 1A, but not the XL $\alpha$ s, regions. Bg, Bg/ll; S, Sacll; F, Fspl, N, NgoMIV; A, Ascl; Bs, BssHII; P, Pstl; Mat, maternal allele; Pat, paternal allele. 
a

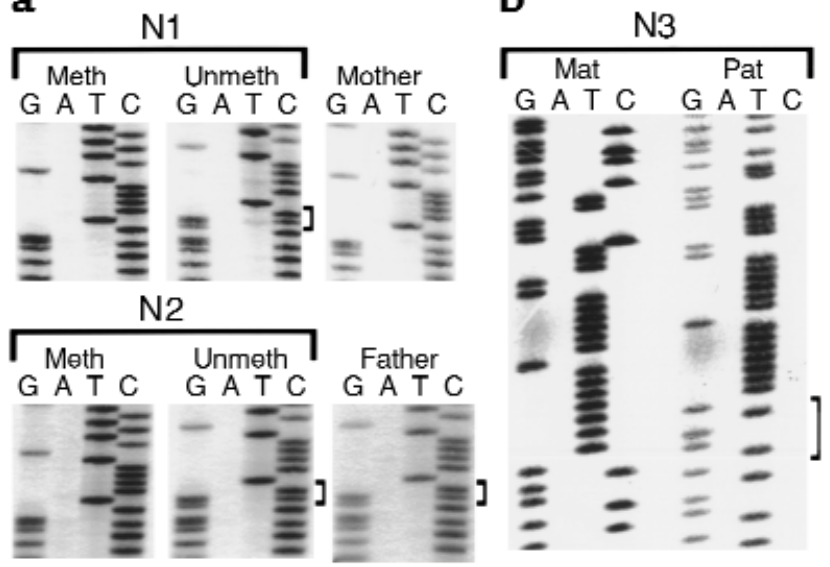

Figure 2

The GNAS1 exon 1A region is methylated only on the maternal allele in normal subjects. (a) Genomic DNA from two normal subjects heterozygous for a 5-bp insertion polymorphism in GNAS1 exon 1A (N1 and N2) was digested with Pstl and Ascl, and fractions containing the 2.8-kb methylated (Meth) and 1.6-kb unmethylated (Unmeth) fragments were purified from agarose gels. The fractions were genotyped for the exon 1A polymorphism using PCR and direct sequencing and for parental assignment were run next to the same reaction performed on genomic DNA from a homozygous parent (mother for $\mathrm{N} 1$, father for N2). The presence of the polymorphic 5-bp insertion is indicated with brackets (although there is compression on these gels, bisulfite-modified genomic sequencing, shown in $\mathbf{b}$, which removed these compressions, confirmed that the insertion is in fact 5 -bp long). For both N1 and N2, the genotypes of methylated and unmethylated fragments corresponded to those of the mother and father, respectively. Identical results were also obtained for N3, the sister of N2 (data not shown). (b) Results of bisulfite-modified genomic sequencing of $\mathrm{N} 3$, showing the methylated maternal allele on the left and the unmethylated paternal allele (which includes the 5-bp insertion polymorphism) on the right. In the paternal allele, all $\mathrm{Cs}$ are unmethylated and are therefore converted to Ts. In the maternal allele all Cs within CpG dinucleotides (eight shown in figure; 25 total in sequencing reaction) are methylated and therefore remain as $\mathrm{Cs}$ (Cs that are not within CpG dinucleotides are unmethylated and are therefore converted to Ts).

nested primers to amplify the sense strand: $5^{\prime}$ TAGGGGT(C/T)GTTGTTATGGGT- $3^{\prime}$ and 5'-AATAACAAAAATCTATTTACCCTCAAAC- $3^{\prime}$ for the first PCR, and $5^{\prime}$-GGTTG(C/T)GTTAGGTGGTTGGT- $3^{\prime}$ and $5^{\prime}$ CTCCTTAATTTAACTCTTAAACAC- $3^{\prime}$ for the second PCR. The PCR products were subcloned into pCRII/TOPO by TA cloning (Invitrogen, Carlsbad, California, USA), and subclones were sequenced using the upstream nested primer.

RT-PCR. RNA was isolated from patient blood samples using the QIAamp RNA Blood Mini kit (QIAGEN). RT-PCR was performed on $1 \mu \mathrm{g}$ total RNA per sample using a protocol described previously (25). The PCR cycling profile consisted of an initial 4minute denaturation at $95^{\circ} \mathrm{C}$, followed by 30 cycles of annealing $\left(56^{\circ} \mathrm{C}, 30\right.$ seconds), extension $\left(72^{\circ} \mathrm{C}, 60\right.$ seconds), and denaturation $\left(95^{\circ} \mathrm{C}, 30\right.$ seconds), with a final cycle with a 10-minute extension. The follow- ing sets of upstream and downstream PCR primers were used: $5^{\prime}$-CTTAACGCCCACCACCGCTC- $3^{\prime}$ and $5^{\prime}$ TTCGCAACTTGAGAGCGTGCAGACGC- $3^{\prime}$ for NESP55 (9); 5'-CGTCGACAACGGCTCCGGCATGTGCAAAGC-3' and $5^{\prime}$-AATAGTGATGACCTGGCCGTCAGGCAGCTC-3' for $\beta$-actin (26); and $5^{\prime}$-CTGCGTCAGGTGGCTGGC-3' and $5^{\prime}$-TTTACCAGATTCTCCAGCAC-3' for the exon 1A transcript (the latter primer is complementary to exon 2). RT-PCR reactions without added reverse transcriptase were performed in parallel to rule out DNA contamination. RT-PCR reactions were run on $6 \%$ acrylamide gels. Specific RT-PCR products were isolated using the QIAEX II kit (QIAGEN) and directly sequenced or were subcloned into PCRII/TOPO by TA cloning (Invitrogen). Individual clones were genotyped by sequencing.

Genomic sequencing. Sequence of the exon $1 \mathrm{~A}$ upstream region of GNAS1 was obtained from clone $309 f 20$ of the RPCI1 human PAC library (GenBank accession no. AF246983).

\section{Results}

The exon $1 \mathrm{~A}$ region is imprinted in bumans. To evaluate the methylation of the exon 1A region in humans, a probe spanning the homologous region of GNAS1 was generated by PCR and hybridized with leukocyte genomic DNA digested with PstI alone, or PstI plus either of the methylation-sensitive enzymes AscI or NgoMIV. In all samples, digestion with $P s t \mathrm{I}$ alone produced the expected 2.8-kb band (Figure 1b, right panel). In samples from 19 normal subjects (including four unaffected relatives of PHPIB patients) and three AHO patients, digestion with PstI plus either the methylation-sensitive enzymes AscI or NgoMIV, produced both the 2.8-kb and smaller bands resulting from digestion by the methylation-sensitive enzymes, consistent with the presence of one methylated and one unmethylated allele. We identified a 5-bp insertion polymorphism within exon $1 \mathrm{~A}$ that we used to determine which parental allele is methylated. Genomic DNA from three informative normal subjects was digested with PstI and AscI, and the regions containing the $2.8-\mathrm{kb}$ methylated and $1.6-\mathrm{kb}$ unmethylated bands were gel purified. Genotyping of each fraction for the polymorphism by PCR and direct sequencing demonstrated that in humans, as in mice, the maternal allele is methylated and the paternal allele is unmethylated (Figure 2a, data not shown).

Maternal-specific methylation of the exon 1A region was confirmed by bisulfite-modified genomic sequencing. Bisulfite treatment of genomic DNA mutates unmethylated cytidines to uracils, whereas methylated cytidines (located within CPG dinucleotides) remain unmodified. After subsequent PCR, unmethylated cytidines are converted to thymine $(\mathrm{T})$, whereas methylated cytidines remain as cytidine (C). Individual PCR products were subcloned and sequenced. The parental origin of each product can be determined by the presence or absence of the 5-bp insertion polymorphism. In the example shown in Figure 2b, N3 is the normal sister 
Figure 3

RT-PCR analysis of blood RNA. (a) RT-PCR was performed on total RNA isolated from blood of two normal subjects (N1 and N2) and two PHPIB patients (patients 1 and 3 ) using exon $1 \mathrm{~A}$-specific upstream and exon 2-specific downstream primers. Direct sequencing of RT-PCR products is shown above with the position of the polymorphic 5-bp insertion indicated by brackets. For N1 and N2, only RT-PCR products with the 5-bp insertion are identified (which for both N1 and N2 is the paternal allele; see Figure 2a). In contrast, RT-PCR products both with and without the polymorphic 5-bp insertion are amplified from patients 1 and 3 , indicating biallelic expression of exon $1 \mathrm{~A}$ mRNAs in these patients. Below each sequence are results of genotyping of subcloned products. For patient 1 , clones with the 5-bp insertion are derived from the paternal allele, whereas for patient 3 these clones are derived from the maternal allele. (b) RT-PCR was performed on total RNA isolated from blood of normal subjects $(\mathrm{N})$ and four PHPIB patients (patients $1,5,8$, and 12) using NESP55-specific primers (above) or $\beta$-actin-specific primers (below). In patients 1, 5, and 8 both GNAS1 alleles are methylated in the NESP55 upstream region, whereas in patient 12 only one allele is methylated in this region (see Table 1). The presence or absence of enzyme in the RT reaction is shown above each lane.

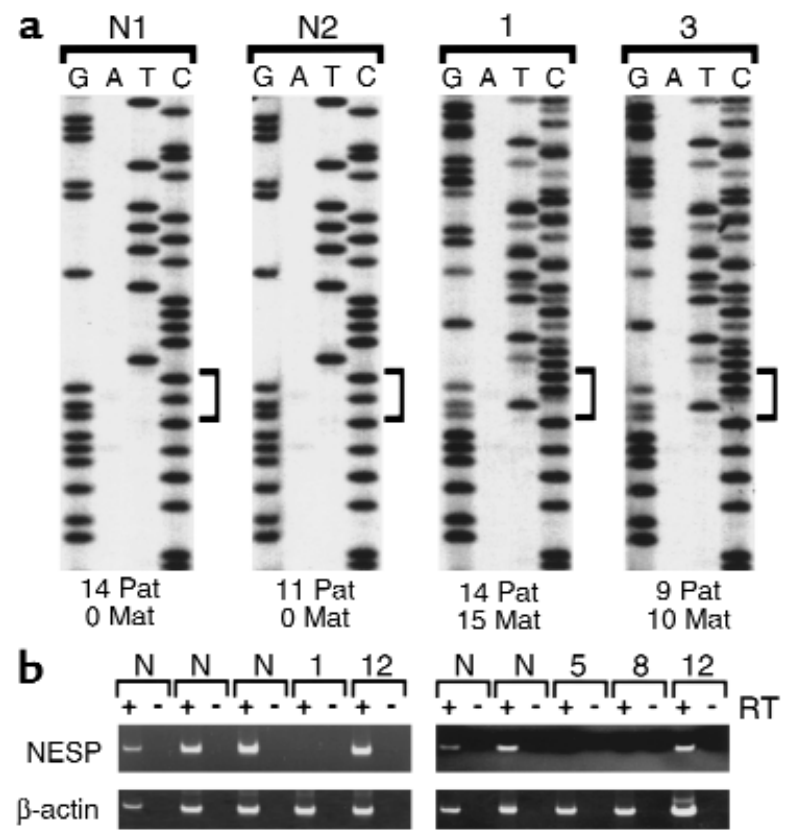

have a paternal-specific methylation pattern (being unmethylated) within the exon $1 \mathrm{~A}$ region. Deletion of the maternal copy of the GNAS1 exon 1A region was ruled out in these patients either by the presence of heterozygosity for the exon 1A polymorphism or by quantitative Southern analysis (data not shown).

To determine the allele-specific expression of exon $1 \mathrm{~A}$ mRNA transcripts in PHPIB patients, RT-PCR was performed with exon 1A-specific primers on RNA samples maternal allele in humans.

To examine allele-specific expression of exon 1A mRNA transcripts, RT-PCR was performed with exon $1 \mathrm{~A}$-specific primers on blood total RNA samples from two normal subjects (N1, N2) who were informative for the exon $1 \mathrm{~A}$ polymorphism. Direct sequencing revealed that exon $1 \mathrm{~A}$ mRNA is only expressed from the paternal allele in the two normal subjects (Figure 3a; in both $\mathrm{N} 1$ and $\mathrm{N} 2$ the paternal allele has the polymorphic 5-bp insertion). This was confirmed by sequencing of subcloned products that showed all were derived from the paternal allele. Therefore, as in mice, exon 1A mRNA is normally expressed only from the paternal allele in humans.

The exon $1 A$ region has a paternal-specific imprinting pattern on both alleles in PHPIB. In contrast to the results obtained from normal or AHO samples, the exon $1 \mathrm{~A}$ region was completely digested by both AscI or NgoMIV in all 13 PHPIB patients who were studied (Figure 1b, right panel; Table 1). These results indicate that in all PHPIB patients both alleles
Table 1

GNAS1 methylation analysis in PHPIB patients ${ }^{\mathrm{A}}$

\begin{tabular}{|c|c|c|c|c|c|}
\hline \multirow[b]{2}{*}{ Patient } & \multirow[b]{2}{*}{ Familial $^{\mathrm{B}}$} & \multirow[b]{2}{*}{ Bone disease ${ }^{C}$} & \multicolumn{3}{|c|}{ Methylation } \\
\hline & & & NESP55 & XLOs & $1 \mathrm{~A}$ \\
\hline 1 & $-\mathrm{D}$ & - & $\mathrm{CH}_{3} / \mathrm{CH}_{3}{ }^{\mathrm{E}}$ & $-1-$ & $-1-$ \\
\hline 2 & - & - & $\mathrm{CH}_{3} / \mathrm{CH}_{3}$ & $-1-$ & $-1-$ \\
\hline & Yes & - & $-/ \mathrm{CH}_{3}$ & $\mathrm{CH}_{3} /-$ & $-/-$ \\
\hline 4 & Yes & - & $-/ \mathrm{CH}_{3}$ & $\mathrm{CH}_{3} /-$ & $-1-$ \\
\hline 5 & - & Yes & $\mathrm{CH}_{3} / \mathrm{CH}_{3}$ & $\mathrm{CH}_{3} /-$ & $-1-$ \\
\hline 6 & - & - & $-/ \mathrm{CH}_{3}$ & $\mathrm{CH}_{3} /-$ & $-/-$ \\
\hline 7 & - & - & $\mathrm{CH}_{3} / \mathrm{CH}_{3}$ & $\mathrm{CH}_{3} /-$ & $-1-$ \\
\hline 8 & - & - & $\mathrm{CH}_{3} / \mathrm{CH}_{3}$ & $\mathrm{CH}_{3} /-$ & $-/-$ \\
\hline 9 & - & - & $-/ \mathrm{CH}_{3}$ & $\mathrm{CH}_{3} /-$ & $-1-$ \\
\hline 10 & - & Yes & $-/ \mathrm{CH}_{3}$ & $\mathrm{CH}_{3} /-$ & $-1-$ \\
\hline 11 & - & - & $-/ \mathrm{CH}_{3}$ & $\mathrm{CH}_{3} /-$ & $-/-$ \\
\hline 12 & - & - & $-/ \mathrm{CH}_{3}$ & $\mathrm{CH}_{3} /-$ & $-1-$ \\
\hline 13 & - & - & $-/ \mathrm{CH}_{3}$ & $\mathrm{CH}_{3} /-$ & $-1-$ \\
\hline \multicolumn{3}{|c|}{ Normal subjects } & $-/ \mathrm{CH}_{3}(14)$ & $\mathrm{CH}_{3} /-(14)$ & $\mathrm{CH}_{3} /-(15)$ \\
\hline \multicolumn{3}{|c|}{ Unaffected family members } & & & $\mathrm{CH}_{3} /-(4)^{\mathrm{F}}$ \\
\hline \multicolumn{3}{|c|}{ AHO patients } & $-/ \mathrm{CH}_{3}(1)$ & $\mathrm{CH}_{3} /-(1)$ & $\mathrm{CH}_{3} /-(3)$ \\
\hline
\end{tabular}

AResults of GNAS1 methylation analysis in the regions containing the NESP55, XLas, and exon $1 \mathrm{~A}$ promoter and first exons in 13 PHPIB patients. The number of normal subjects and AHO patients studied for each region is shown in parentheses. ${ }^{B}$ Defined as the presence of at least one other affected family member. ${ }^{C}$ Defined as the presence of osteitis fibrosa cystica and severely elevated alkaline phosphatase at presentation. DLack of familial or bone disease is indicated with a dash. EMethylation for each allele is shown as maternal/paternal; $\mathrm{CH}_{3}$, methylated; -, unmethylated. Allele-specific methylation of the NESP55 and XL $\alpha$ s promoters in normal subjects has been determined by others previously $(8,9)$. FIncludes the two unaffected parents of patient 1 , the unaffected brother of patient 6 , and the unaffected father of patient 11. 


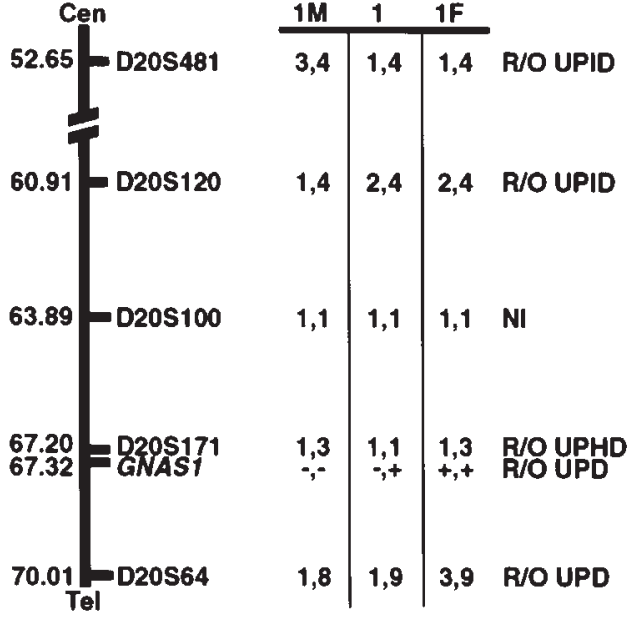

Figure 4

Genotyping of 20q polymorphic markers in PHPIB patient 1 and her parents. The relative position of each marker in megabases is indicated on the left (http://cedar.genetics.soton.ac.uk/public_html/) from centromere (Cen) to telomere (Tel). Results and interpretation for each marker are shown on the right. The GNAS1 polymorphism is a 5 -bp insertion within exon $1 \mathrm{~A}$ ( + and - indicates presence or absence of the insertion, respectively). NI, not informative; R/O UPD, rule out uniparental disomy; R/O UPID, rule out uniparental isodisomy; R/O UPHD, rule out uniparental heterodisomy. (UPID is inheritance of two copies of the same chromosome from a single parent; UPHD is inheritance of one copy each of both chromosomes from a single parent).

from two PHPIB patients (patients 1 and 3 ) who were informative for the exon 1A polymorphism (Figure 3a). Direct sequencing of the RT-PCR products revealed the presence of transcripts both with and without the polymorphic 5-bp insertion, indicating that exon $1 \mathrm{~A}$ mRNAs are expressed from both parental alleles. This was confirmed by genotyping of individually subcloned products, which showed similar numbers of cloned products derived from the maternal and paternal alleles, respectively (Figure 3a). Parallel reactions performed without reverse transcriptase in the RT reaction generated no PCR product, ruling out the presence of DNA contamination in the reaction (data not shown). Biallelic expression of exon 1A mRNA is consistent with the exon $1 \mathrm{~A}$ promoter being unmethylated in both alleles. Analysis of the XLOS- and NESP55-promoter regions in PHPIB. Southern analysis with an XL $\alpha$ s-specific probe (Figure 1b, middle panel; Table 1) demonstrated partial digestion of one AHO and 14 normal samples by each of two methylation-sensitive enzymes (AscI and $B s s \mathrm{HII}$ ), consistent with the presence of one methylated maternal and one unmethylated paternal allele (as previously described, ref. 8). Similar results were obtained in 11 of 13 PHPIB patients (Table 1). In two samples the DNA was completely digested by AscI and Bss HII, consistent with a paternal-specific (unmethylated) XLos methylation pattern in both alleles (Figure $1 \mathrm{~b}$, Table 1). The lack of an XLos imprinting defect in the majority of PHPIB patients suggests that neither the XLos-promoter region nor its gene product are important for the pathogenesis of PHPIB.
Similar analysis of the NESP55-promoter region (Figure 1b, left panel; Table 1) demonstrated partial digestion of both normal and AHO DNA samples by each of three methylation-sensitive enzymes (SacII, FspI, NgoMIV), consistent with the presence of one methylated paternal and one unmethylated maternal allele (as previously described, ref. 9). This same pattern was observed in only eight of 13 PHPIB patients (Table 1), while DNA from the other five patients was resistant to digestion by all three methylation-sensitive enzymes, consistent with both alleles being methylated (Figure $1 \mathrm{~b}$, Table 1). Therefore, a subset of PHPIB patients has a paternal-specific NESP55 methylation pattern on both alleles. The lack of a NESP55 imprinting defect in the majority of PHPIB patients suggests that neither the NESP55-promoter region nor its gene product are important for the pathogenesis of PHPIB.

RT-PCR of blood RNA with NESP55-specific primers showed that NESP55 mRNA was expressed in normal patients and in a PHPIB patient with normal NESP55promoter methylation, but was not expressed in the three patients that we examined in whom the NESP55 promoter is methylated on both alleles (Figure 3b). Presumably, both NESP55 alleles are suppressed by promoter methylation. There is no evidence for partial or tissue-specific imprinting of NESP55 (ref. 9; and J. Liu and L.S. Weinstein, unpublished data). These findings suggest that loss of NESP55 expression in blood (and possibly loss of NESP55 expression in all tissues) is not associated with an obvious phenotype.

In two of 13 patients (patients 1 and 2) both alleles had a paternal-specific methylation pattern within all three GNAS1 imprinted regions. Methylation was normal in both unaffected parents of patient 1 (Figure 1b). In patient 1, paternal uniparental disomy (UPD) or deletion of the GNAS1 maternal allele was ruled out by genotyping of $20 \mathrm{q}$ markers in patient 1 and her parents (Figure 4) and by demonstration that patient 1 was heterozygous for the exon 1A-insertion polymorphism. (UPD is the inheritance of both copies of a chromosome or chromosomal region from one parent). Both parents of patient 2 are unavailable for genotyping. We have not formally ruled out deletion of the maternal copy of GNAS1 in patient 2, but we would expect this to lead to PHPIA, rather than PHPIB. Of the remaining 11 patients, three are also abnormally methylated at the NESP55- but not at the XLos-promoter region, while eight are normally methylated at both the NESP55 and XLos promoters (Figure 1b, Table 1). Normal methylation of the XLas region effectively rules out UPD in all other patients. In the three patients with abnormal NESP55- and exon 1A-promoter methylation, deletion is unlikely because one would have to invoke two separate deletions on either side of the XLos promoter.

\section{Discussion}

We have shown that PHPIB is commonly (or possibly always) associated with a GNAS1 imprinting defect leading to a paternal-specific imprinting pattern on both 
alleles. Whereas most cases of PHPIB appear to be sporadic, a significant number of cases are familial. Moreover, a small subset of PHPIB patients present with severe osteitis fibrosa cystica due to the effects of elevated circulating PTH on bone (pseudohypohyperparathyroidism; refs. 27-29). It was therefore unclear whether PHPIB is a single genetic entity or whether the various clinical and genetic presentations of PHPIB resulted from genetic defects at distinct loci. The presence of a common GNAS1 methylation defect in all PHPIB patients in our cohort, including both sporadic and familial cases and in those who present with overt bone disease (Table 1), suggests that all forms of PHPIB are caused by a common defect involving the GNAS1 locus. Measurement of GNAS1 exon 1A methylation may be a useful diagnostic tool for the evaluation of patients who present with PTH resistance.

While paternal UPD of $20 \mathrm{q}$ could produce the defects observed in PHPIB, it was detected in none of 12 patients in whom this could be assessed. These data suggest that UPD is not commonly associated with this disease, although we hypothesize that paternal UPD may be a rare cause of PHPIB. In most cases, PHPIB most likely results from failure to switch from the paternal to maternal imprint in the oocyte, and this could explain why the phenotype was only inherited maternally in four PHPIB families (4). Linkage of PHPIB to $20 q 13$ (4) suggests that, at least in familial cases, the imprinting defect is due to a mutation near or within the GNAS1 locus. We speculate that mutations might reside within the exon $1 \mathrm{~A} D \mathrm{DMR}$ and are presently searching for such mutations in our patient cohort.

While the imprinted NESP55 (30) or XL $\alpha$ s (11) promoters have been implicated in PHPIB, the lack of a NESP5 or XL $\alpha$ s-promoter defect in the majority of PHPIB patients suggests that neither promoter nor their gene products are important for the pathogenesis of PHPIB. The presence of paternal-specific imprinting of the NESP55 and XL $\alpha$ s promoters on both alleles in some patients suggests that the NESP55, XLQs, and exon $1 \mathrm{~A}$ regions may share common elements that are required for the establishment and maintenance of their imprinting. However, our findings indicate that the three GNAS1 imprinted regions are not always coregulated, since in most cases paternal-specific methylation of exon 1A is not associated with similar changes in the NESP55- and XL $\alpha$ s-promoter regions. Likewise, paternal-specific methylation of the NESP55 promoter is not always associated with similar changes in XLos. A paternalspecific antisense transcript that originates just upstream of the XL $\alpha$ s promoter may be important for NESP55 imprinting $(31,32)$.

The most likely explanation for PTH resistance in PHPIB is markedly decreased $G_{s} \alpha$ expression in renal proximal tubules due to both GNAS1 alleles having a paternal-specific imprinting pattern. Abnormal imprinting would have no affect on $\mathrm{G}_{\mathrm{s}} \alpha$ expression in other tissues where it is normally expressed from both alleles. This would explain why $\mathrm{G}_{\mathrm{s}} \alpha$ expression in blood cells is unaffected in PHPIB (1) and, perhaps, why the AHO phenotype is absent in these patients. In contrast, maternal or paternal GNAS1 mutations produce $G_{s} \alpha$ haploinsufficiency in these same tissues (33, 34), and this might explain the AHO phenotype that is common to both PHPIA and PPHP patients.

The close proximity of the exon 1 A region to the $G_{s} \alpha$ promoter and its abnormal methylation in PHPIB patients suggest that this region is critical for $G_{s} \alpha$ imprinting. However, $\mathrm{G}_{\mathrm{s}} \alpha$ imprinting is tissue-specific, while the maternal-specific methylation of the exon $1 \mathrm{~A}$ DMR is widespread (e.g., present in blood cells). Therefore, any mechanism to explain the tissue-specific imprinting of $\mathrm{G}_{\mathrm{s}} \alpha$ must involve an interaction of the DMR with a tissue-specific factor. One potential mechanism for imprinting of $G_{s} \alpha$ could involve reciprocal regulation of the exon $1 \mathrm{~A}$ and $\mathrm{G}_{\mathrm{s}} \alpha$ promoters in the paternal allele, due to promoter competition or negative regulation of the $G_{s} \alpha$ promoter by exon $1 A$ transcripts. This model would predict that expression of exon $1 \mathrm{~A}$ mRNAs would be limited to tissues in which $G_{s} \alpha$ is imprinted. However, we have shown in mice that exon $1 \mathrm{~A}$ and $\mathrm{G}_{\mathrm{s}} \alpha \mathrm{mRNAs}$ are expressed in a similar distribution pattern (10), and thus we believe that this mechanism is unlikely. Alternatively, the exon $1 \mathrm{~A}$ DMR could have a binding site for a repressor that is specifically expressed in renal proximal tubules. Such a repressor would bind to its cognate site in the paternal allele, but be prevented from binding to the maternal allele because the site is methylated (Figure 5a). Another potential mechanism invokes boundary elements with-

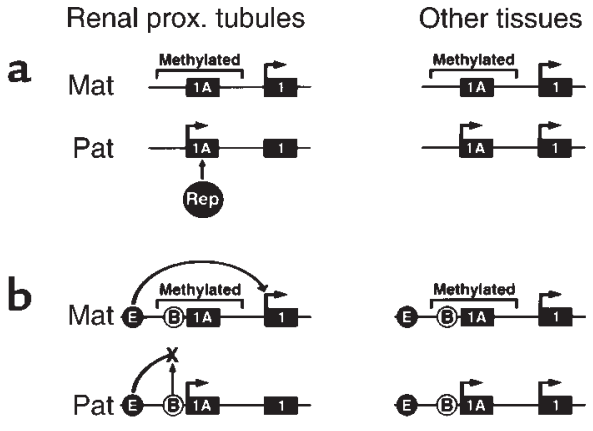

\section{Figure 5}

Potential mechanisms for tissue-specific imprinting of $G_{s} \alpha$. (a) The exon 1A DMR may contain a binding site for a tissue-specific repressor (Rep) that binds to the paternal allele (Pat) in renal proximal tubules, leading to silencing of the $G_{s} \alpha$ (exon 1) promoter. Binding to the maternal allele (Mat) is prevented by methylation of the binding site. The model predicts that the repressor is expressed in renal proximal tubules, but not in other tissues where $\mathrm{G}_{\mathrm{s}} \alpha$ is biallelically expressed. (b) The exon 1A DMR may contain one or more boundary elements (B) that block activation of the $\mathrm{G}_{\mathrm{s}} \alpha$ promoter by an upstream enhancer $(E)$ in the paternal allele, but do not block enhancer-promoter interactions in the maternal allele due to methylation. This is the mechanism by which lgf2 is imprinted $(36,37)$. This model predicts that expression of $\mathrm{G}_{\mathrm{s}} \alpha$ in other tissues is not dependent on the upstream enhancer. 
in the exon 1A DMR that block activation of the $G_{s} \alpha$ promoter by an upstream enhancer in the paternal allele. In the maternal allele the boundary elements would not block promoter activation because they are methylated (Figure 5b). This is the mechanism by which the paternally methylated DMR located upstream of $H 19$ leads to paternal-specific expression of Igf2 (35). Recently the H19 DMR was shown to contain binding sites for CTCF, a protein that binds to mammalian boundary elements $(36,37)$. Binding of CTCF to the H19 DMR in the paternal allele is inhibited by methylation of its binding sites. A boundary element model predicts that expression of $\mathrm{G}_{\mathrm{s}} \alpha$ in other tissues is not dependent on the upstream enhancer. Further studies in PHPIB patients and in mice with deletions of the Gnas imprinted regions will help determine if and how these regions regulate each other and the mechanism by which $G_{s} \alpha$ is imprinted in a tissue-specific manner.

\section{Acknowledgments}

We thank M. Reitman and A. Spiegel for critical reviewing of the manuscript, R. Vinitsky for technical assistance, and the staff of the NIH Intramural Sequencing Center for genomic sequencing.

1. Levine, M.A., et al. 1983. Resistance to multiple hormones in patients with pseudohypoparathyroidism. Association with deficient activity of guanine nucleotide regulatory protein. Am. J. Med. 74:545-556.

2. Schipani, E., et al. 1995. Pseudohypoparathyroidism type Ib is not caused by mutations in the coding exons of the human parathyroid hormone (PTH)/PTH-related peptide receptor gene. J. Clin. Endocrinol. Metab. 80:1611-1621.

3. Jobert, A.S., et al. 1998. Absence of functional receptors for parathyroid hormone and parathyroid hormone-related peptide in Blomstrand chondrodysplasia. J. Clin. Invest. 102:34-40.

4. Jüppner, H., et al. 1998. The gene responsible for pseudohypoparathyroidism type Ib is paternally imprinted and maps in four unrelated kindreds to chromosome 20q13.3. Proc. Natl. Acad. Sci. USA. 95:11798-11803.

5. Weinstein, L.S., and Yu, S. 1999. The role of genomic imprinting of $\mathrm{G}_{\mathrm{s}} \alpha$ in the pathogenesis of Albright hereditary osteodystrophy. Trends Endocrinol. Metab. 10:81-85.

6. Silve, C., Santora, A., Breslau, N., Moses, A., and Spiegel, A. 1986. Selective resistance to parathyroid hormone in cultured skin fibroblasts from patients with pseudohypoparathyroidism type Ib. J. Clin. Endocrinol. Metab. 62:640-644.

7. Kozasa, T., Itoh, H., Tsukamoto, T., and Kaziro, Y. 1988. Isolation and characterization of the human Gs alpha gene. Proc. Natl. Acad. Sci. USA. 85:2081-2085.

8. Hayward, B.E., et al. 1998. The human GNAS1 gene is imprinted and encodes distinct paternally and biallelically expressed $G$ proteins. Proc. Natl. Acad. Sci. USA. 95:10038-10043.

9. Hayward, B.E., Moran, V., Strain, L., and Bonthron, D.T. 1998. Bidirectional imprinting of a single gene: GNAS1 encodes maternally, paternally, and biallelically derived proteins. Proc. Natl. Acad. Sci. USA. 95:15475-15480

10. Liu, J., Yu, S., Litman, D., Chen, W., and Weinstein, L.S. 2000. Identification of a methylation imprint mark within the mouse Gnas locus. Mol. Cell. Biol. 20:5808-5817.

11. Peters, J., et al. 1999. A cluster of oppositely imprinted transcripts at the Gnas locus in the distal imprinting region of mouse chromosome 2. Proc. Natl. Acad. Sci. USA. 96:3830-3835.

12. Swaroop, A., Agarwal, N., Gruen, J.R., Bick, D., and Weissman, S.M. 1991.
Differential expression of novel Gs $\alpha$ signal transduction protein cDNA species. Nucleic Acids Res. 19:4725-4729.

13. Ishikawa, Y., Bianchi, C., Nadal-Ginard, B., and Homcy, C.J. 1990. Alternative promoter and $5^{\prime}$ exon generate a novel $\mathrm{G}_{\mathrm{s}} \alpha \mathrm{mRNA}$. J. Biol. Chem. 265:8458-8462.

14. Constancia, M., Pickard, B., Kelsey, G., and Reik, W. 1998. Imprinting mechanisms. Genome Res. 8:881-900.

15. Bartolomei, M.S., and Tilghman, S.M. 1997. Genomic imprinting in mammals. Annu. Rev. Genet. 31:493-525.

16. Tilghman, S.M. 1999. The sins of the fathers and mothers: genomic imprinting in mammalian development. Cell. 96:185-193.

17. Patten, J.L., et al. 1990. Mutation in the gene encoding the stimulatory $G$ protein of adenylate cyclase in Albright's hereditary osteodystrophy. N. Engl. J. Med. 322:1412-1419.

18. Weinstein, L.S., et al. 1990. Mutations of the $\mathrm{G}_{\mathrm{s}} \alpha$-subunit gene in Albright hereditary osteodystrophy detected by denaturing gradient gel electrophoresis. Proc. Natl. Acad. Sci. USA. 87:8287-8290.

19. Weinstein, L.S. 1998. Albright hereditary osteodystrophy, pseudohypoparathyroidism and $\mathrm{G}_{\mathrm{s}}$ deficiency. In $\mathrm{G}$ proteins, receptors, and disease. A.M. Spiegel, editor. Humana Press. Totowa, New Jersey, USA. 23-56.

20. Levine, M.A., Jap, T.S., Mauseth, R.S., Downs, R.W., and Spiegel, A.M. 1986. Activity of the stimulatory guanine nucleotide-binding protein is reduced in erythrocytes from patients with pseudohypoparathyroidism and pseudopseudohypoparathyroidism: biochemical, endocrine, and genetic analysis of Albright's hereditary osteodystrophy in six kindreds. J. Clin. Endocrinol. Metab. 62:497-502.

21. Davies, S.J., and Hughes, H.E. 1993. Imprinting in Albright's hereditary osteodystrophy. J. Med. Genet. 30:101-103.

22. Campbell, R., Gosden, C.M., and Bonthron, D.T. 1994. Parental origin of transcription from the human GNAS1 gene. J. Med. Genet. 31:607-614.

23. Yu, S., et al. 1998. Variable and tissue-specific hormone resistance in heterotrimeric $\mathrm{G}_{\mathrm{s}}$ protein $\alpha$-subunit $\left(\mathrm{G}_{\mathrm{s}} \alpha\right)$ knockout mice is due to tissuespecific imprinting of the $\mathrm{G}_{\mathrm{s}} \alpha$ gene. Proc. Natl. Acad. Sci. USA. 95:8715-8720.

24. Yu, S., et al. 2000. Paternal versus maternal transmission of a stimulatory $G$ protein $\alpha$ subunit knockout produces opposite effects on energy metabolism. J. Clin. Invest. 105:615-623.

25. Warner, D.R., Gejman, P.V., Collins, R.M., and Weinstein, L.S. 1997. A novel mutation adjacent to the switch III domain of $\mathrm{G}_{\mathrm{s} \alpha}$ in a patient with pseudohypoparathyroidism. Mol. Endocrinol. 11:1718-1727.

26. Tokunaga, K., Taniguchi, H., Yoda, K., Shimizu, M., and Sakiyama, S. 1986. Nucleotide sequence of a full-length cDNA for mouse cytoskeletal $\beta$-actin mRNA. Nucleic Acids Res. 14:2829.

27. Costello, J.M., and Dent, C.E. 1963. Hypo-hyperparathyroidism. Arch. Dis. Child. 38:397-407.

28. Kolb, F.O., and Steinbach, H.L. 1962. Pseudohypoparathyroidism with secondary hyperparathyroidism and osteitis fibrosa. J. Clin. Endocrinol. Metab. 22:59-70.

29. Murray, T.M., et al. 1993. Pseudohypoparathyroidism with osteitis fibrosa cystica: direct demonstration of skeletal responsiveness to parathyroid hormone in cells cultured from bone. J. Bone Miner. Res. 8:83-91.

30. Nicholls, R.D. 2000. The impact of genomic imprinting for neurobehavioral and developmental disorders. J. Clin. Invest. 105:413-418.

31. Hayward, B.E., and Bonthron, D.T. 2000. An imprinted antisense transcript at the human GNAS1 locus. Hum. Mol. Genet. 9:835-841.

32. Wroe, S.F., et al. 2000. An imprinted transcript, antisense to Nesp, adds complexity to the cluster of imprinted genes at the mouse Gnas locus. Proc. Natl. Acad. Sci. USA. 97:3342-3346.

33. Levine, M.A., et al. 1980. Deficient activity of guanine nucleotide regulatory protein in erythrocytes from patients with pseudohypoparathyroidism. Biochem. Biophys. Res. Commun. 94:1319-1324.

34. Farfel, Z., Brickman, A.S., Kaslow, H.R., Brothers, V.M., and Bourne, H.R. 1980. Defect of receptor-cyclase coupling protein in pseudohypoparathyroidism. N. Engl. J. Med. 303:237-242.

35. Schmidt, J.V., Levorse, J.M., and Tilghman, S.M. 1999. Enhancer competition between $H 19$ and Igf 2 does not mediate their imprinting. Proc. Natl. Acad. Sci. USA. 96:9733-9738.

36. Bell, A.C., and Felsenfeld, G. 2000. Methylation of a CTCF-dependent boundary controls imprinted expression of the Igf2 gene. Nature. 405:482-485.

37. Hark, A.T., et al. 2000. CTCF mediates methylation-sensitive enhancerblocking activity at the H19/Igf2 locus. Nature. 405:486-489. 